\title{
Patrimonialisation de la pâture
}

Entre marginalisation et valeur universelle

Recognition of grazing heritage through legislation. Between marginalization and universal value

\section{Olivier Barrière}

\section{(2) OpenEdition Journals}

Édition électronique

URL : https://journals.openedition.org/tc/7486

DOI : $10.4000 /$ tc. 7486

ISBN : 0248-6016

ISSN : 1952-420X

Éditeur

Éditions de l'EHESS

\section{Édition imprimée}

Date de publication : 1 mai 2015

Pagination : 182-201

ISBN : 9782713224874

ISSN : 0248-6016

Référence électronique

Olivier Barrière, "Patrimonialisation de la pâture », Techniques \& Culture [En ligne], 63 | 2015, mis en ligne le 16 décembre 2018, consulté le 29 septembre 2022. URL : http://journals.openedition.org/tc/ 7486 ; DOI : https://doi.org/10.4000/tc.7486 


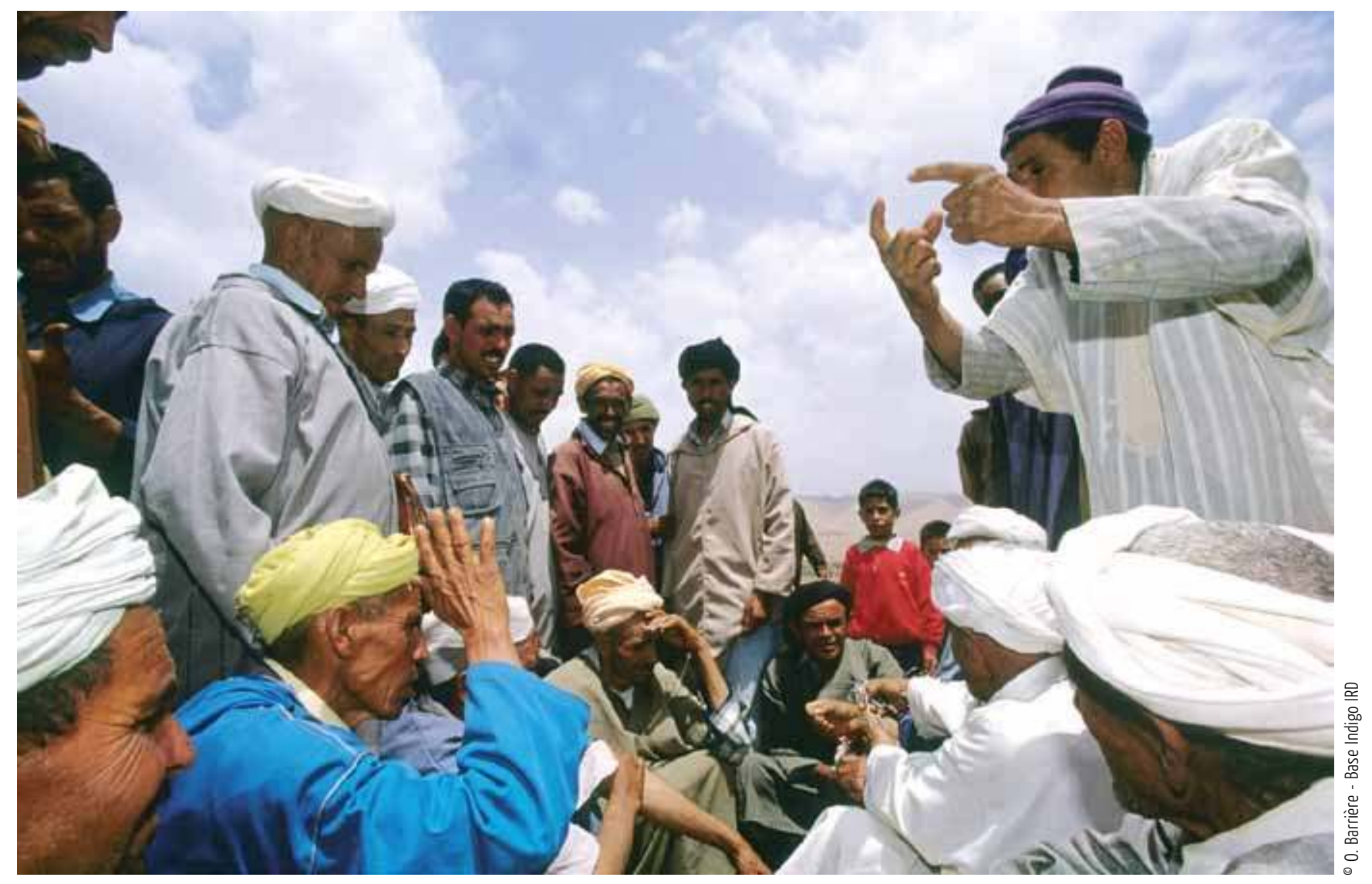




\section{PATRIMONIALISATION DE LA PÂTURE}

\section{Entre marginalisation et valeur universelle}

La contrainte foncière devient souvent une impasse pour l'activité pastorale face au régime du droit de propriété. En effet, l'accès à la ressource herbe (sur pied) ${ }^{1}$ se voit de plus en plus contrariée à la fois par la compétition des usages sur l'espace que l'on retrouve dans les différents terrains étudiés dans ce Thema (urbanisation, activités diverses, mises en défens) et le besoin de pâturages de dimensions adaptés aux troupeaux dont les effectifs ont souvent

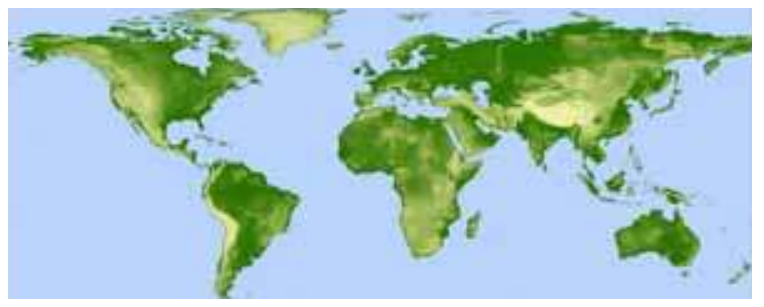
eu tendance à croître. « Aller là où se trouve l'herbe » définit le pastoralisme par la mobilité qu'il induit. Le souci constant se situe ainsi dans une quête incessante de la pâture, qu'elle soit généralement horizontale ou verticale. La mobilité dans l'espace suit une dynamique à la fois spatiale et temporelle, particulièrement pour l'élevage ovin qui constitue ici notre référent: transhumer (« changer de terre ») du haut vers le bas et vice versa selon la saison et la disponibilité alimentaire. Mais la mobilité, estivale et hivernale, n'est pas toujours constante, l'agnelage impose une immobilisation. Et c'est surtout, du moins en Europe du sud, l'absence de pâture hivernale, d'un accès à l'herbe naturelle qui condamne le troupeau dans la bergerie, hormis des logiques purement économiques (coût du gardiennage par exemple). Le stock de fourrage s'impose, qu'il soit produit par l'éleveur lui-même ou acheté. Dans le premier cas, un espace foncier constitué de champs mis en culture est nécessaire pour répondre à l'enjeu de « l'autonomie fourragère ». En définitive, l'accès à l'herbe définit bien le facteur limitant de l'activité pastorale. Cet accès est conditionné par une relation « foncière », c'est-à-dire $\mathrm{au}$ « fonds » (portion d'espace délimité), qui lui, est objet d'un droit d'appropriation dans 


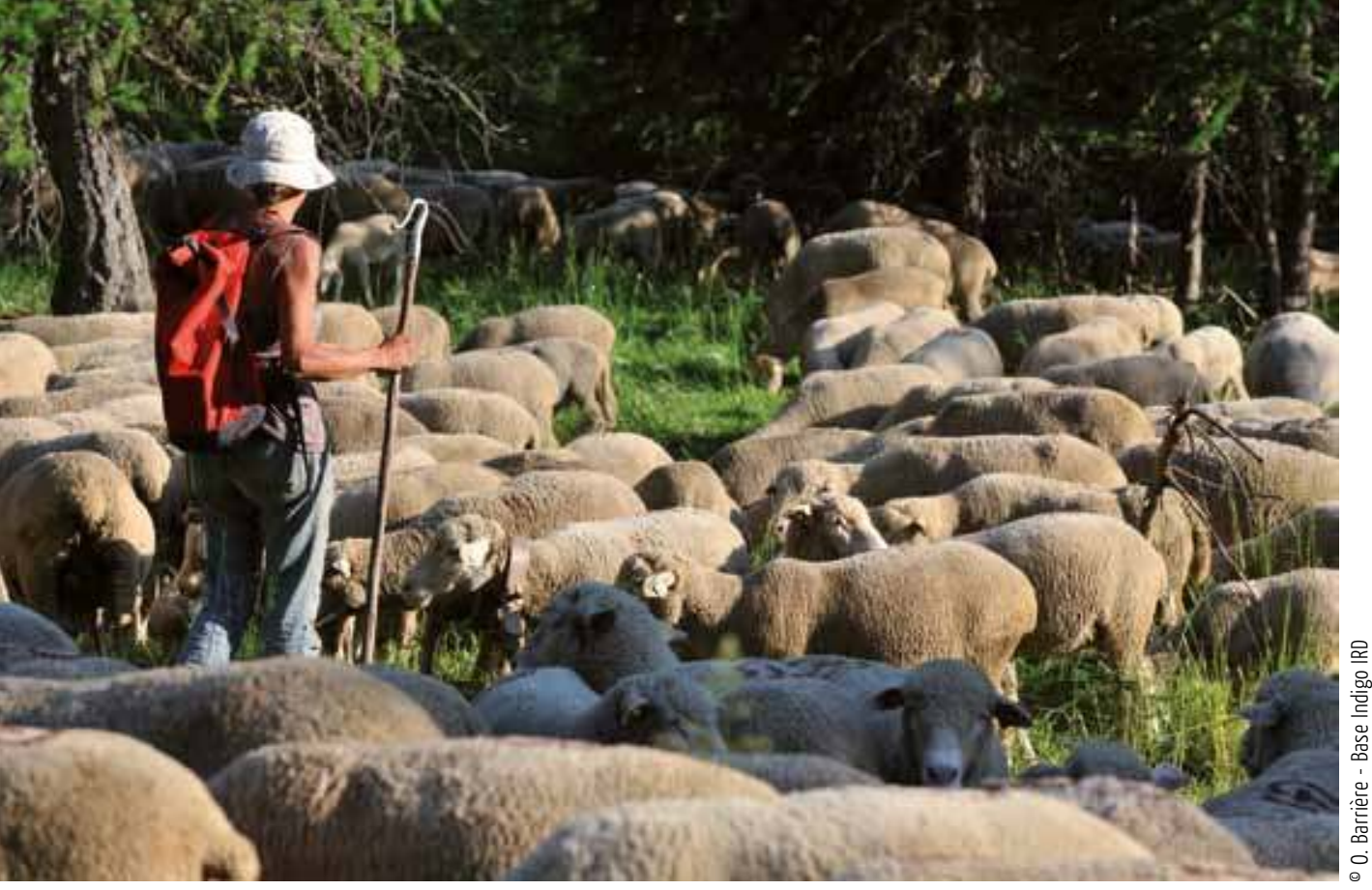

Bergère

dans les Alpes de Haute Provence

Garde estivale sous bois, Allos / Mont Pelat, 2012. tie d'une façon intrinsèque de l'histoire des territoires. En effet, l'élevage extensif intègre autant le paysage que l'agriculture et la foresterie dans les constructions territoriales. Cependant, partout dans le monde, la mobilité de cette forme d'élevage expose souvent un dérangement d'ordre politique par une «insaisissabilité » des acteurs éleveurs dû au mouvement souvent constant, à la fois pour les États et les sédentaires. Les droits des populations transhumantes sont défendus par le droit international notamment par la Déclaration de Dana (Jordanie) 7 avril 2002, sur les populations mobiles et la protection de l'environnement, et la Déclaration des Nations Unies sur les droits des peuples autochtones (2007). Certains pays adoptent des codes pastoraux ou pour le moins une réglementation spécifique.

Prenons quelques exemples du continent africain et de la Mongolie. La loi du 27 février 2001 portant Charte pastorale en République du Mali. Novateur, ce texte n'est pas la périphrase d'une loi occidentale et les auteurs sont bien africains. Il innove d'une part, en ce qu'il, reconnaît pleinement l'activité pastorale en précisant les droits essentiels des pasteurs, notamment en matière de mobilité des animaux et d'accès aux ressources pastorales; et d'autre part, la charte confère aux pasteurs une responsabilité environnementale en termes d'obligations qui leur incombent particulièrement dans l'exercice des activités pastorales. La Mauritanie adopte le 26 juillet 2000 une loi portant code pastoral dont l'objectif est la « gestion rationnelle de l'espace pastoral », les parcours relevant du domaine de l'État, dans le but de préserver et de promouvoir le pastoralisme pour « une évolution harmonieuse du développement rural ». Très conscient de l'importance économique, sociale et culturelle de l'activité pastorale, le législateur offre une reconnaissance légale du pastoralisme dans sa mobilité (art.2 \& 3) et dans ses droits sur les ressources pastorales (eau, végétation). Celles-ci endossent le statut de "ressources communes", qui restent cependant appropriées par la Nation. Dans ce texte, l'espace pastoral, affecté exclusivement à l'activité pastorale (art.13), relève d'un régime domanial « collectif » inaliénable, imprescriptible et dont l'appropriation (privative) est considérée comme illégale (art.14). Le rédacteur semble bien penser ici à un domaine public pastoral dans 
7 mars 2014, Maroc Haut Atlas

versant sud

Amazir « TAYARTE », tribu Aït Sedrat, vallée qui traverse le douar Ait Hammou dans l'Oued Dades

Le statut juridique importe peu à la brebis qui broute ici

des xérophytes épineux, mais l'accès à la ressource est essentiel à l'éleveur qui conduit le troupeau en fonction du droit dont il bénéficie.

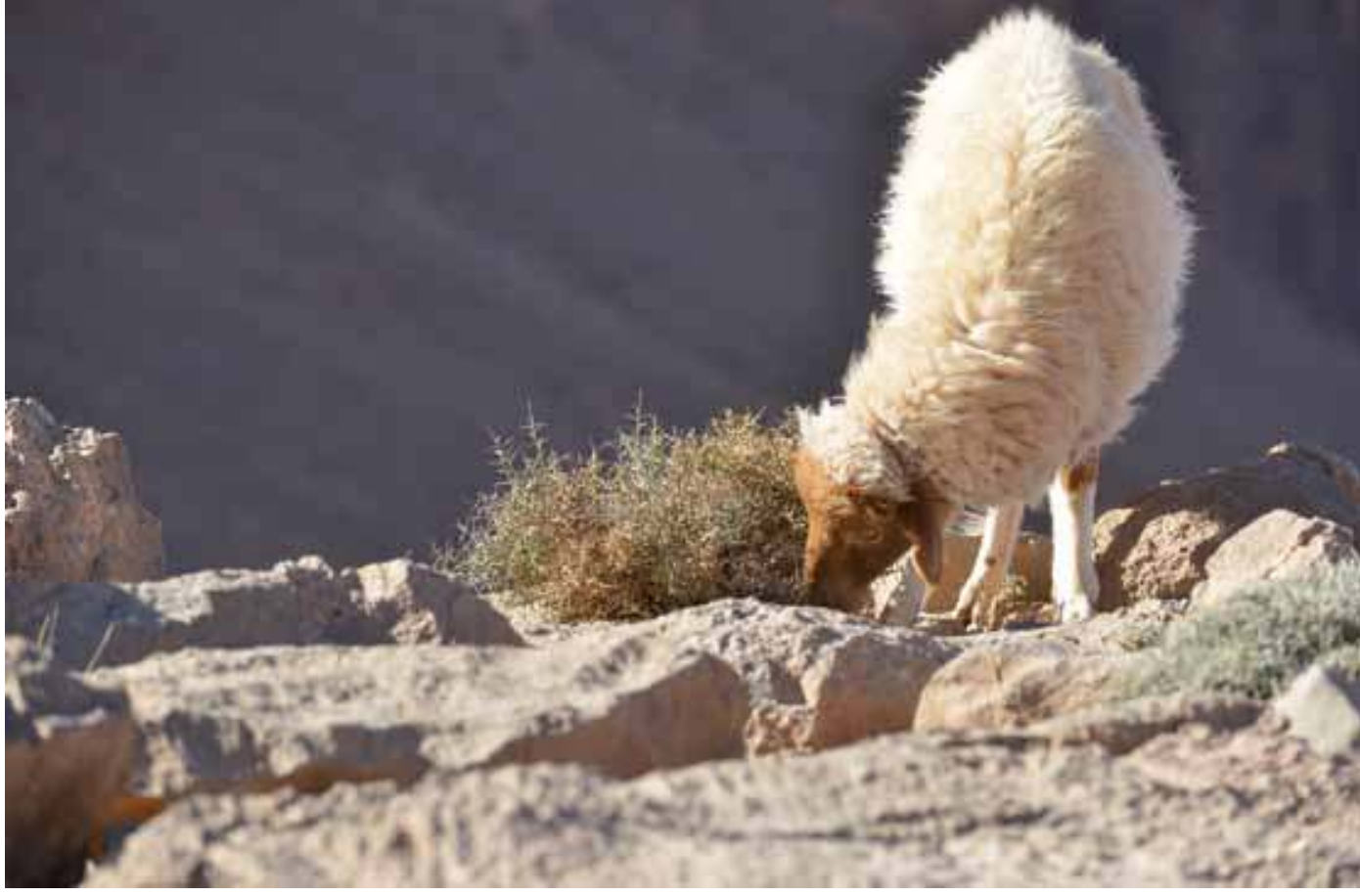

0. Barrière - Base Indigo IRD

le but de conférer à cet espace une protection maximale. Mais le décret d'application de cette loi, toujours attendu, devrait préciser les conditions de sa mise en œuvre. Le Niger adopte une législation sur le pastoralisme par l'ordonnance du 2 mars 1993 fixant les principes d'orientation du Code rural (article 23 à 39). Les droits des pasteurs sur l'espace sont définis, notamment par la notion de terroirs d'attache pastoraux et de délimitation des couloirs de passage pour les animaux.

Enfin en Mongolie, si l'enjeu d'une approche territoriale et intégrée est particulièrement soulignée ${ }^{3}$, le passage brutal à l'économie de marché en 1990 donne lieu à la suppression des systèmes coutumiers. La mobilité des troupeaux se réduit considérablement. Ladoption d'une loi foncière en 1994, puis en 2002, organise des accès libres au parcours l'été et l'automne avec des pouvoirs accor-

Delta intérieur du fleuve Niger (Mali), Région de Mopti, Jworo de Wuro Neema

A. Sidibe, garde son petit troupeau de brebis et surveille en même temps la zone dont l'accès est strictement controlée, 1993 (Région de Mopti). dés aux autorités locales pour limiter la charge pastorale et l'accès aux terres salées faisant entrer la dynamique pastorale dans une contractualisation (bail sur les pâturages). Si la réglementation et les pratiques s'opposent au développement du régime de la propriété privée, l'attribution et la gestion des pâturages restent non résolue. Les droits

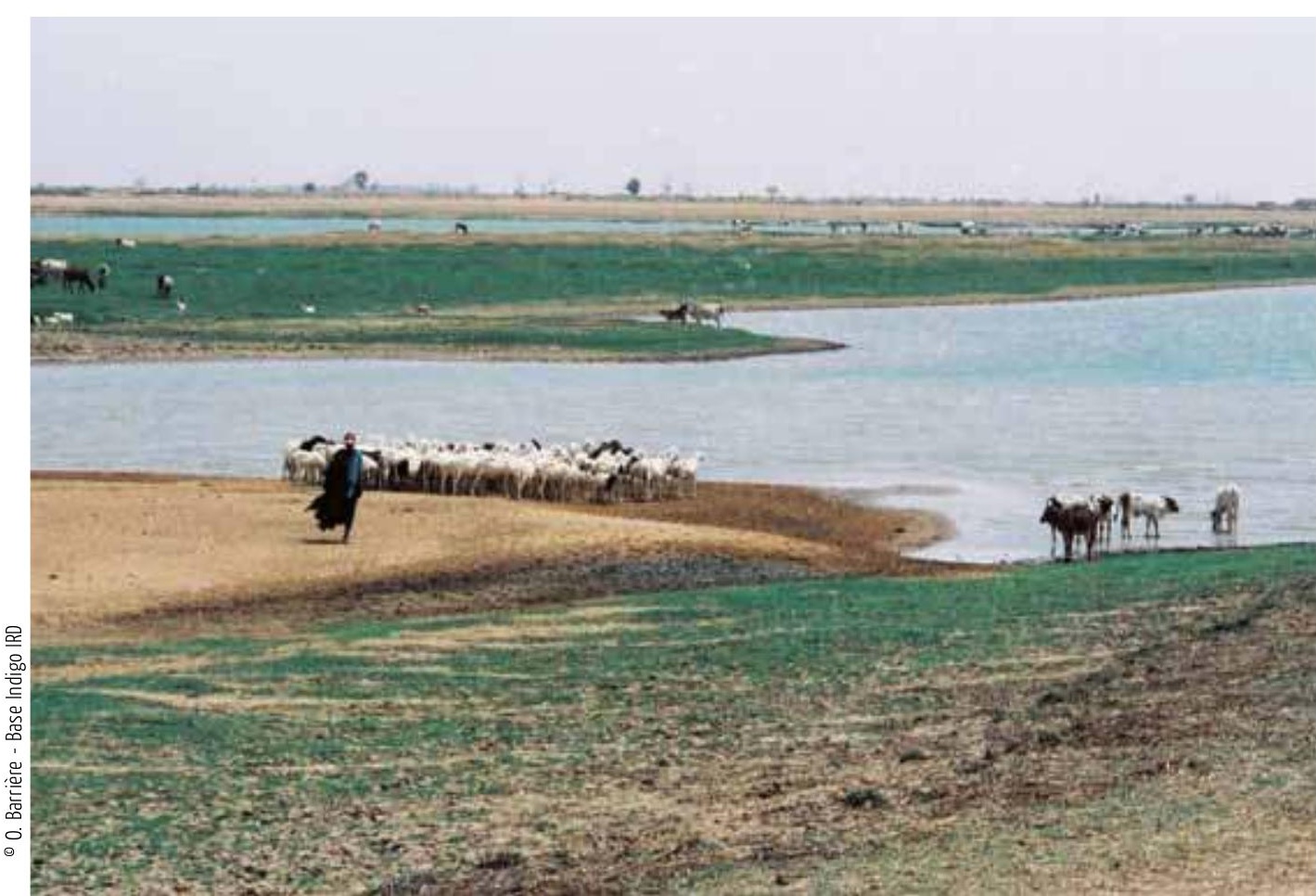


sortirons par la reconnaissance mondiale d'une culture pastorale vivante conduisant à dégager des alternatives à l'appropriation exclusive et quasi-absolue qui définit le régime de la propriété foncière.

Avouons-le de suite, le régime de la propriété foncière conduit au diktat d'un seul, qu'il soit privé, public ou collectif. La pâture se trouve de ce fait inféodée au pouvoir exclusif et absolu du propriétaire. Ainsi s'impose le passage obligé de la contractualisation, du bon vouloir d'un intérêt particulier (du fonds approprié) sur les autres, voire de l'intérêt général puisqu'en France, en zone de montagne le pâturage revêt, par la loi, ce caractère. Le rapport objectivé à l'espace fractionné en « biens » (chose marchande) conduit souvent à une forme d'impasse, par la contrainte de la relation marchande de l'appropriation. Il en résulte une réduction de l'espace à une mosaïque foncière de propriétés avec des propriétaires souvent divers et multiples (ce qui s'oppose à la réalité d'Amérique latine du Latifundia). La conséquence majeure qui en découle s'exprime par une opposition entre l'individuel, le patrimoine personnel, et le collectif, un besoin rattaché au territoire, constitutif d'un véritable patrimoine commun des acteurs de ce dernier.

Luniversalité de cette dualité sévit sur l'ensemble des continents, avec des tailles de parcelles bien différentes selon les régions du monde. Au Maroc par exemple, la question se pose sur le foncier « collectif » dépendant de groupes « sociaux-ethniques » que sont les tribus disposant d'une personnalité juridique et d'un territoire reconnus par le législateur. Par définition, le collectif s'oppose au privé. De cette trivialité, ce qui concentre l'attention porte sur la relation entre la mise en culture et le maintien des parcours. L'enjeu pastoral est directement concerné par la compétition à l'accès foncier qui fait l'objet de pressions et de relations tendues. Une tension

\section{Près de l'amazir « TAYARTE », tribu Ait Sedrat (douar Ait Hammou au fond de la vallée de l'Oued Dades)}

Le berger est dans cet amazir (cabane) depuis 2 mois,

il a prévu de se déplacer pour d’autres pâturages dans les 15 jours. La sécheresse se fait sentir ce mois de mars alors quill pleut sur Ouarzazate. La région est décrétée sinistrée par les autorités, ce qui lui permettra d'obtenir des apports alimentaires pour son troupeau.

Ce berger nous avoue que depuis dix ans surtout il constate un véritable changement du climat/7 mars 2014.

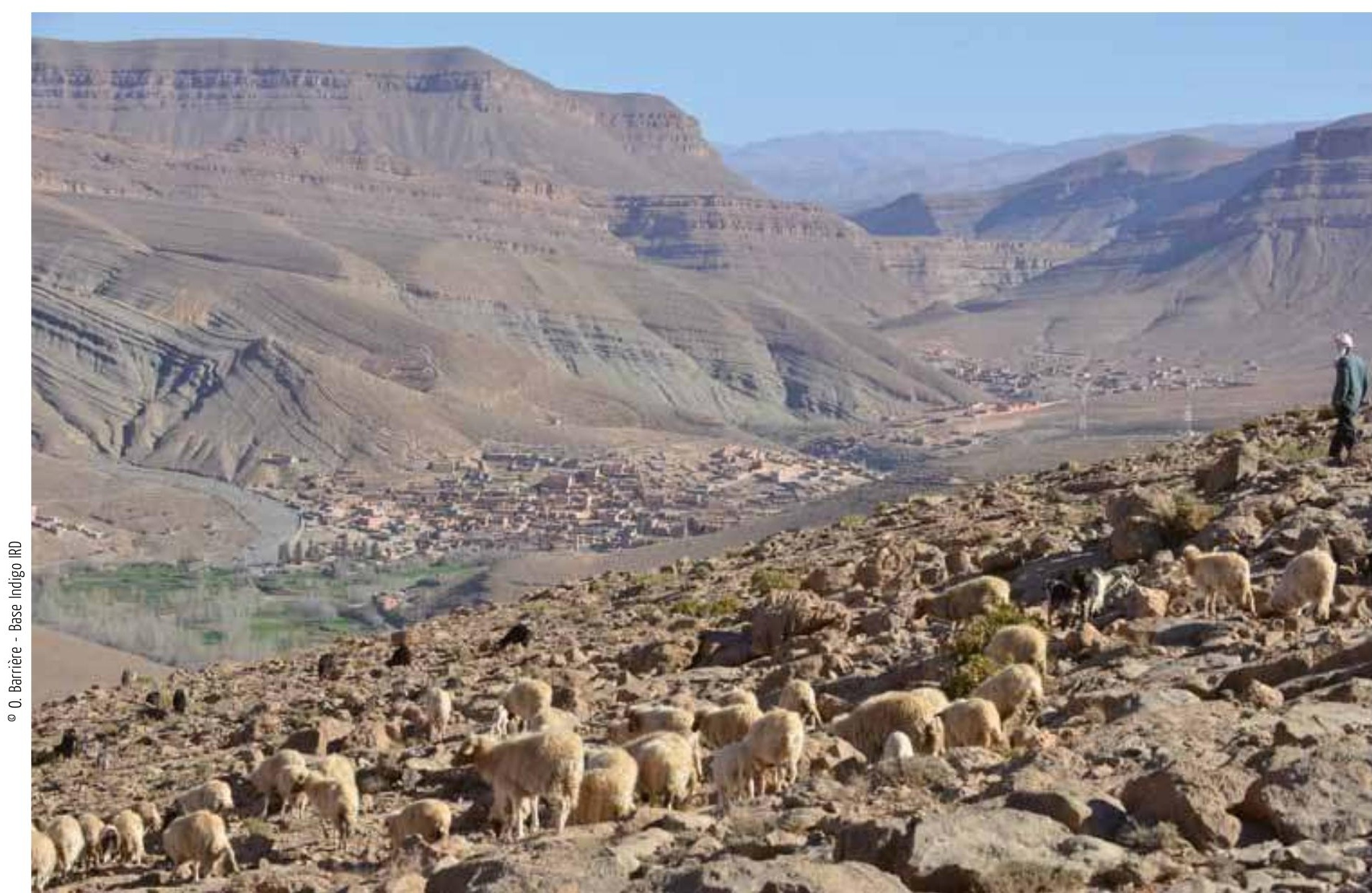




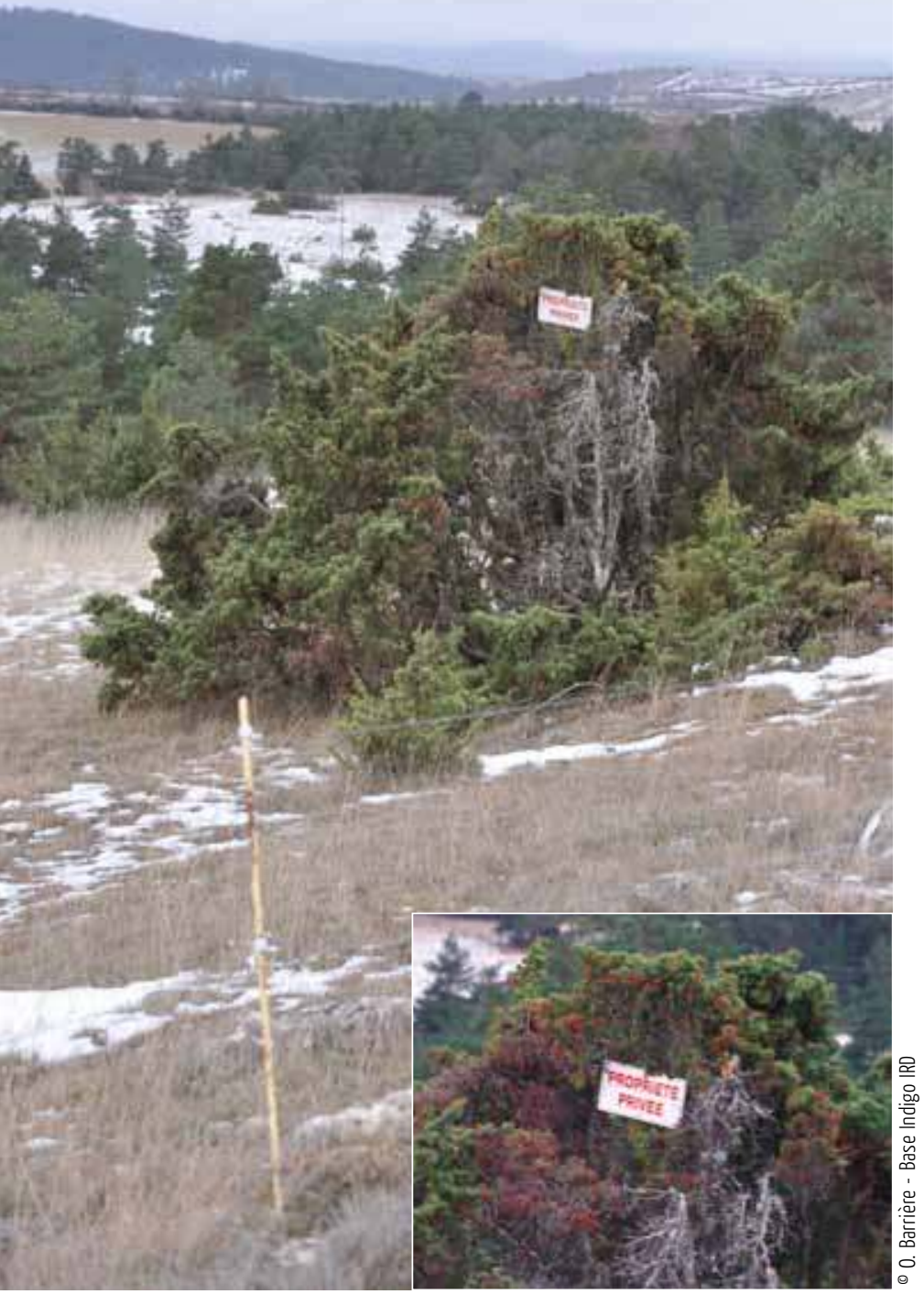

Propriété privée

Sur le Causse noir,

entre Lanuéjols et Veyreau,

la marque de l'appropriation foncière

se manifeste dans le paysage

au moyen de clôtures pour les

troupeaux mais aussi de temps

en temps par des panneaux

revendicatifs « propriété privée »,

une façon d'en interdire explicitement

(juridiquement) l'entrée. d'autant plus accentuée par les aléas climatiques de plus en plus prononcés, exprimant un changement climatique que les prévisions du GIEC (5ème rapport, 2014) soulignent particulièrement dans ces zones circumsahariennes.

La question foncière renvoie à celle de la place du pastoralisme dans les territoires, une question quasiment universelle là où existe un élevage extensif. La raison en est qu'elle porte sur les contours précis entre le droit d'usage (droit d'accès limité à la ressource, en l'occurrence pastorale) et le droit de jouissance (droit de propriété sur le fonds). Ce temps de la relation (juridique) entre l'usage et la jouissance s'ouvre sur un second temps, faisant office de porte de sortie, celui de l'enjeu territorial. Ainsi, nous allons voir qu'en droit, le pâturage se donne en pâture au champ foncier qui s'en saisit en le soumettant souvent au régime, considéré comme inéluctable, de la propriété (I). Mais ce dernier se voit de plus en plus « contraint » au territoire (II).

\section{La pâture soumise au droit de propriété: une marginalisation foncière du pastoralisme}

Dans le rapport foncier la relation à l'espace pastoral dépasse une logique purement agraire. En effet, la ressource pastorale porte davantage sur l'herbe que la terre elle-même, support de cette ressource ${ }^{4}$. Cette production avec ou sans intervention humaine directe définit la ressource appelée « pastorale», qui est consommée sur place par le bétail. De plus, le «terrain » n'est pas continuellement couvert d'herbes en raison du cycle biologique, des saisons, des espèces et de la façon dont les hommes l'investissent. L'espace pastoral est celui utilisé à la pâture des troupeaux avec le plus souvent une idée d'impermanence ou de temporalité et de mouvements cycliques (Meuret \& al. 1995). L'emprise n'est donc pas uniquement celle du sillon de la charrue, ou d'une artificialisation par manipulation du substrat du sol, de la couche supérieure. Cet aspect va conditionner le rapport juridique de l'homme à la terre. De la forme de l'emprise sur l'espace naît le droit sur le « fonds $»^{5}$.

Ce droit foncier exprime en premier lieu un pouvoir sur les hommes. Ces derniers rattachés à un espace le transforment en « territoire », traduction d'un espace socialisé. Le contrôle social garantit une protection dont celle de l'accès aux ressources foncières et environnementales. La maîtrise du pouvoir politique offre une sécurité foncière pour les hommes qui sont soumis à ce pouvoir-là. L'exemple du régime féodal orchestre une 
superposition de droits sur un même espace sur la base de deux domaines: le domaine éminent (droit politique) définit le contrôle territorial, le domaine utile (droit sur la ressource) porte sur les fonctionnalités qu'offre le territoire (culture, coupe de bois, pâture, cueillette...). Le régime étatique est celui qui garantit la propriété foncière, prenant naissance dans le droit romain.

La compréhension du rapport foncier se décline à la fois diachroniquement, dans l'histoire des sociétés et des institutions, et synchroniquement, entre des paradigmes divergents, occidental et celui qui ne l'est pas. Les deux tiers de l'humanité n'évoluant pas sous le régime du droit des biens (Le Roy, 2011 : 348), il est difficilement concevable d'universaliser une seule logique juridique foncière. En d'autres termes, tout ne se décline pas en « droit ou droits de propriété ».

À ce stade deux questions se posent : celle de l'assise juridique du droit foncier pastoral et celle de sa matérialisation. Ces questions émergent en raison du fait que l'emprise pastorale sur la terre (sur le fonds) ne relève pas d'une nature agraire, d'une mise en culture (assortie de labours souvent), mais d'une nature moins dépendante du sol que de l'herbe qui en sort. Cette caractéristique première fait du pastoralisme une activité marginalisée dans l'espace, objet d'affectations voire d'allocations assorties de droits d'appropriation mais également de droits sur le contrôle et l'usage des utilités des ressources territoriales.

L'histoire du droit de la propriété foncière offre un premier éclairage sur la place du pastoralisme. L'origine de ce droit découle de la possession avant que la loi ne le formalise en «propriété ». En effet, la propriété est née de la reconnaissance légale de l'emprise totale du détenteur sur la chose. Le fait de la détention, défendue par la force, s'est commué en droit de propriété par la garantie sociale d'un droit d'aliénation (transférer ou détruire) d'une chose: «... les lois ne protègent pas seulement la propriété, ce sont elles qui la font naître, qui la déterminent, qui lui donnent le rang et l'étendue qu'elle occupe dans les droits du citoyen » (Laboulaye, 1839, 59). La propriété naît donc de la loi qui reconnaît le droit d'abuser (le droit de propriété n'existe qu'avec le droit d'abuser, sinon il n'y a simplement que le droit de possession). Sans loi, pas de propriété: « ce qui est contemporain de la première société, c'est l'occupation momentanée, ou la possession en commun : la propriété ne vient que plus tard, par le progrès des libertés et la lente élaboration des lois » (Proudhon, $1824: 71$ ).

L'enjeu de la propriété du code civil, dans l'histoire du droit de propriété, se définit par le rejet des éléments grevant le fonds (droits multiples) pour le libérer et assurer une souveraineté totale à l'unique propriétaire. Mais là où le régime de la propriété n'a pas émergé d'un processus historique, comme en Occident, les États doivent l'inventer et le créer de toutes pièces en supprimant ce qui peut s'y opposer (le droit interne aux groupes sociaux, dit droit coutumier). Apparaissent ainsi soit un processus de purge dans les législations, soit l'enracinement d'une « domanialisation » de l'espace (domaine national, domaine public, domaine forestier de l'État, domaine des collectivités territoriales ou domaine privé de l'État).

Le passage à la propriété réclame une individualisation du droit sur la terre, condition nécessaire pour parvenir à l'immatriculation, ou enregistrement foncier (art.2511-4 du code civil). Cette procédure transite par celle de la constatation d'une « vivification des terres » (mise en valeur, concept clef dans le droit colonial et de nombreuses législations africaines). L'enjeu des législateurs des États africains indépendants est d'asseoir la propriété foncière en l'instituant au moyen d'un titre, d'un livre ou d'un registre foncier, d'un cadastre, en reléguant au statut de « droit coutumier » le paradigme local du rapport 
à la terre. Le régime domanial quant à lui, particulièrement dans le droit africain, se rapproche du concept de domaine éminent. Et si la sécurisation foncière des exploitants agricoles demeure l'objectif des législateurs, ces derniers la définissent en termes de propriété inscrite dans un plan foncier (genre de cadastre, assorti d'un registre foncier), un livre foncier ou dans un processus d'immatriculation.

Le propre du rapport pastoral à l'espace, au fonds, est de n'y exercer qu'un prélèvement herbacé, au moyen d'une relation de pâture. Et qu'est-ce que la pâture dans le droit? le droit de pacage, c'est-à-dire le droit de faire paître du bétail, touche à la superficie du fonds (la partie qui dépasse que sont les plantations et constructions). La nature juridique de cette prérogative ou du moins de cette activité se définit soit dans un démembrement du droit de propriété, comme droit de jouissance, soit dans un rapport au fonds d'autrui comme un droit d'usage. Dans ce cadre, nous allons approfondir le statut juridique français du pastoralisme, une activité marginalisée dans sa définition foncière, qui se définit d'abord dans la mise en valeur pastorale des territoires avant qu'elle n'aspire à s'intégrer dans le projet de territoire.

\section{Une mise en valeur pastorale des territoires ruraux}

Suite à la loi du 9 juillet 1889 qui abolit le droit de pacage et le droit de parcours, le code civil de 1804 institut le droit de propriété « absolu » (art.544), et une autre mini-révolution intervint dans ce rapport de force entre propriétaires et usagers de l'espace, la loi n ${ }^{\circ} 72-12$ du 3 janvier 1972 relative à la mise en valeur pastorale dans les régions d'économie montagnarde, dite « loi pastorale ». La reconnaissance d'une activité dite « ancestrale » se voit reconnaître pleinement au cœur du hiatus foncier: la relation propriétaires fonciers et éleveurs est enfin formalisée, orchestrée par le regroupement des uns et des autres dans une préoccupation de gestion collective et concertée des espaces pastoraux de montagne. Au sein d'une "association foncière pastorale », les propriétaires d'un périmètre agropastoral sont regroupés pour assurer la « mise en valeur » des terrains agricoles et pastoraux. Les différents éleveurs sont réunis au sein de « groupements pastoraux » dans un souci de mutualisation et d'amélioration de l'exploitation pastorale des espaces. L'outil phare se traduit par ce qu'on appelle une convention pluriannuelle de pâturage, forme souple de relation contractuelle entre le propriétaire du fonds et l'exploitant de l'herbe située sur ce fonds. Le chapitre III du code rural, des articles L113-2 à L113-5 et R113-1 à R113-12, porte explicitement sur la « mise en valeur pastorale » en zone de montagne (définit par le législateur par des handicaps engendrant une vie plus difficile avec la restriction de certaines activités). La transformation progressive de la place du pastoralisme en montagne trouve son origine dans l'évolution même des représentations, traduites par les politiques publiques, que la société, se fait de la montagne et des activités inféodées. Le besoin exprimé de conservation de l'espace naturel, du développement touristique, du développement des économies montagnardes donnera lieu à la loi montagne du 9 janvier 1985 (op.cit.) et à la loi plus récente relative au développement des territoires ruraux, du 23 février 2005 affirmant que l'État est « garant de la solidarité nationale en faveur des territoires ruraux et de montagne et reconnaît leur spécificité ». Apparaît ainsi la notion de solidarité et de territoires locaux, avec le jeu d'échelle entre le national et le local. Mais, mieux encore, ce texte érige la reconnaissance du pastoralisme par l'édification de l'activité pastorale au statut d'intérêt général pour son rôle fondamental à « la 


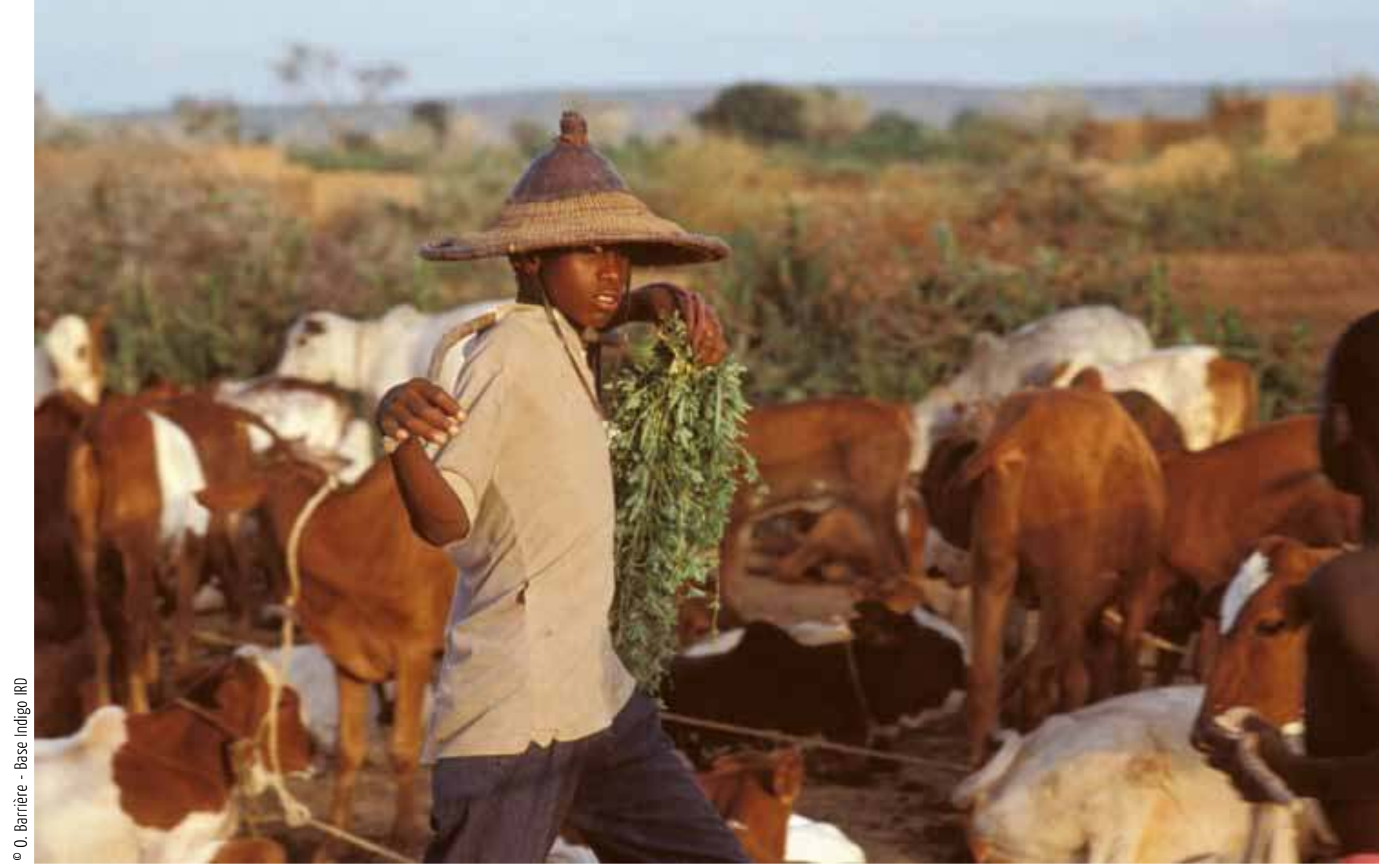

production, à l'emploi, à l'entretien des sols, à la protection des paysages, à la gestion et au développement de la biodiversité ». Enfin une définition légale est donnée au pastoralisme par l'espace concerné, « les pâturages d'utilisation extensives et saisonnières » avec une territorialisation et une fonction à la fois écologique et sociale de l'activité pastorale (art. L113-2 du code rural). Les zones concernées par le pastoralisme sont expressément citées par le législateur: les communes classées en zone de montagne, en plus de celles comprises dans les zones délimitées par le préfet, après avis de la Chambre d'Agriculture.

Les terres de ces zones à vocation pastorale sont susceptibles de faire l'objet de contrats d'exploitation spécifiques (depuis la loi pastorale de 1972 modifiée en 2010): des baux ruraux et des conventions pluriannuelles d'exploitation agricoles ou de pâturage pour une durée de cinq ans et non exclusive d'utilisation du fonds à des fins non agricole (art. L481-1 du code rural). Les propriétaires peuvent également réaliser une convention de mise en exploitation agricole de leurs fonds, pour trois ou six ans.

La circulation des troupeaux fait également l'objet d'une attention particulière par le législateur qui permet au préfet, « lorsque l'état d'abandon ou le défaut d'entretien d'un terrain empêche la circulation des troupeaux », d'accorder à la demande de l'association foncière pastorale ou du groupement pastoral ou, à défaut, des exploitants intéressés, un droit de passage sur ce fonds pour une durée qui ne peut excéder un an, tacitement renouvelable en l'absence d'opposition, après mise en demeure du propriétaire (art. L135-6 du code rural).

Le code forestier, quant à lui, organise la protection de la forêt contre la dent du brouteur. Mais les droits anciens sont préservés, et d'autres créés dans une relation 
contractuelle. La différence doit être faite entre les forêts communales et les forêts de l'État. Ainsi, l'exercice du pâturage dans les forêts privées des communes ou sections de communes soumis au régime forestier est possible aux habitants (sous forme de droit de jouissance) pour le pâturage des bovins, ovins, équidés et porcins à l'exclusion des caprins en raison des dommages qu'ils portent aux arbres dont ils attaquent l'écorce. Dans les forêts de l'État ne sont admis à exercer un droit d'usage quelconque que les habitants dont les droits étaient le 31 juillet 1827 reconnus fondés (par décision de justice ou administrative). Cependant, il ne peut être fait dans les forêts de l'État aucune concession de droit d'usage de quelque nature et sous quelque prétexte que ce soit. En effet, la règle est celle de l'interdiction de principe de conduire en forêt domaniale ou privée ou sur les terrains qui en dépendent, des chèvres ou des moutons, sous peine d'une amende pouvant aller jusqu'à 3750 euros. Ceux qui disposeraient de titre valable pour prétendre bénéficier du pacage pour leurs chèvres ou moutons peuvent réclamer une indemnité du fait de cette interdiction, fixée de gré à gré ou par un tribunal judiciaire. Dans certaines localités toutefois, le préfet peut, par décision spéciale prise sur proposition de l'ingénieur de l'ONF, autoriser le pacage de brebis ou moutons (article L.138-10 du code forestier).

Le droit de pâturage et de panage ${ }^{6}$ en forêt n'est exerçable que dans les cantons déclarés défensables (la forêt « défensable » étant celle qui est en état de se défendre contre les troupeaux qui pâturent), par l'ONF. Chaque année, les maires doivent assurer la publication, dans les communes usagères, des cantons déclarés défensables et du nombre de bestiaux admis au pâturage, qui ont été portés à leur connaissance par l'ONF. S'il y a lieu, ils dressent dans un délai de 15 jours un état de répartition, entre les usagers, du nombre des bestiaux admis à pâturer (article L.138-7 du code forestier). Les usagers ne peuvent jouir de ce droit que pour les bestiaux à leur propre usage et non pour ceux dont ils font commerce, sous peine de se voir infliger une amende pouvant aller jusqu'à 3750 euros. Les droits d'usage dont ceux de pâturage, panage et glandée qui ne peuvent être convertis en cantonnement (hors d'accès), peuvent être rachetés à leur bénéficiaire par le propriétaire de la forêt, moyennant une indemnité. Toutefois, là où l'exercice du droit de pâturage est devenu une absolue nécessité pour les habitants d'une ou plusieurs communes, ces droits ne peuvent faire l'objet d'un rachat.

Faut-il le souligner, l'histoire du rapport forêt/élevage est fait de tensions et de conflits. Entre le monde forestier et celui de l'élevage les relations sont parties d'une absence de concertation que l'évolution législative a contraint à établir par la reconnaissance croissante du pastoralisme. Cependant, les liens entre élevage et territoire se sont construits par étapes. La loi pastorale en 1972 puis la loi montagne en 1985 et la création du CERPAM (Centre d'Études et de Réalisations Pastorales Alpes Méditerranée) en 1977, ont favorisé l'activité pastorale auprès des forestiers qui y trouvent de nos jours un intérêt écologique et financier. Enfin dans les années quatre-vingt-dix le développement des enjeux écologiques (l'ouverture des milieux pour la biodiversité, la lutte contre les incendies) et par conséquence « l'environnementalisation » de l'Office National des Forêts a souligné l'importance du pastoralisme composant essentiel du territoire.

Confrontée au régime du droit de propriété, qui trouve son fondement dans l'histoire de la relation à l'espace, la pâture se voit saisie par une nouvelle territorialité qui se fonde sur le projet territorial. 


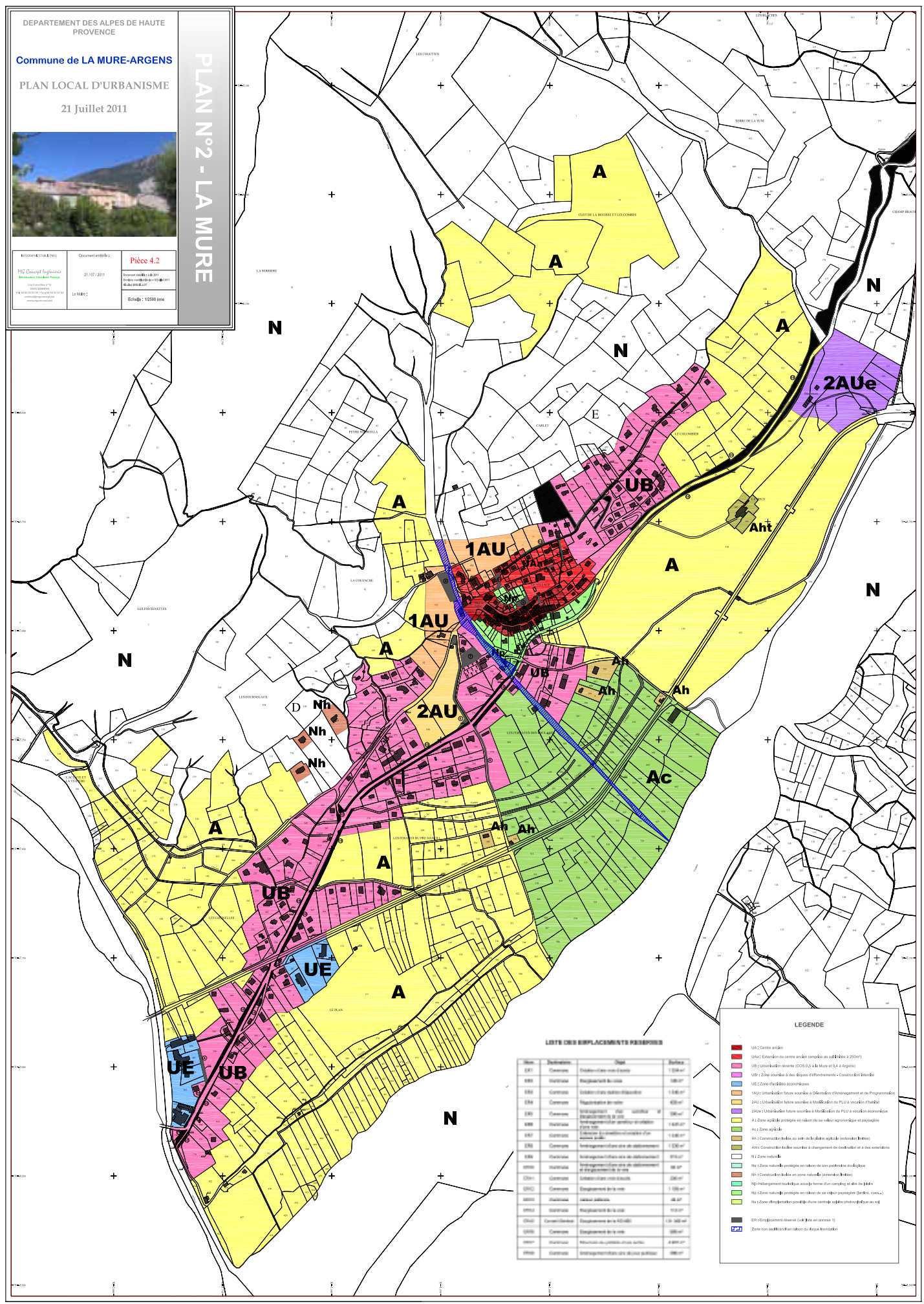

Exemple d'un zonage PLU, commune de Mure-Argens dans les Alpes de HauteProvence

- UB = urbanisation récente

- $U A=$ centre ancien

- $U A a=$ extension du centre ancien

- $U \mathrm{Br} r=$ zone soumise à des risques d'effondrement

- $\mathrm{AU}=$ urbanisation future soumise à ... $\mathrm{A}=$ zone agricole protégée en raison de sas valeur agronomique et paysagère $A c=$ zone agricole $A h$ = construction isolée au sein de plaine agricole (extension limitée)
- $\mathrm{N}=$ zone naturelle

- $\mathrm{Ne}=$ zone naturelle protégée en raison de son patrimoine écologique

- $\mathrm{Nh}=$ construction isolée en zone naturelle (extension limitée)

- $\mathrm{Nl}=$ hébergement touristique

- $\mathrm{Np}$ = zone naturelle protégée en raison de sa valeur paysagère

- Ns = zone d'implantation possible d'une centrale solaire photovoltäqque 


\section{L'intégration du pastoralisme dans les projets de territoire}

À l'échelle de la commune l'outil urbanistique, que nous développer, tel que le plan local d'urbanisme, dont la première vocation est un zonage et un projet de territoire avec le Plan d'aménagement et de développement durable (PADD), l'identification des zones pastorales devrait devenir un enjeu de plus en plus prégnant. Il ne l'est pas encore directement. En effet, le code de l'urbanisme ignore le pastoralisme et ce dernier s'intègre par conséquence dans le zonage $\mathrm{A}$ (agricole), « à protéger en raison du potentiel agronomique biologique ou économique des terres agricoles ». Le zonage « $\mathrm{P} »$ pour « pastoral », n'existant pas et supposant appartenir de facto dans l'agriculture et le naturel (N). Le zonage « pastoral » serait un entre-deux qui n'a pas encore trouvé explicitement sa place dans ces outils.

Le CERPAM a proposé en 2012 la mise en ouvre d'un Plan d'Occupation Pastoral Intercommunal, « POPI » de la Dracénie, située au nord du Var, aux portes du Verdon (7 communes). Les élus locaux souhaitaient connaître l'importance de l'utilisation pastorale du territoire des communes, et l'impact de cette utilisation vis-à-vis d'enjeux de lutte contre les incendies, écologiques ou paysagers. Le POPI est établi à partir de cellules de concertation: élus et éleveurs locaux, administration (DDTM), services techniques et experts (Chambre d'Agriculture du Var, Association des Communes Forestières du Var, Centre Régionaux de la Propriété Forestière, Office National des Forêts, Fédération Départementale des Chasseurs, Conservatoire d'Espaces Naturels, ...). Une réunion annuelle avec l'ensemble des 24 éleveurs concernés permet une mise à jour du plan, et la préparation en concertation avec les élus et les représentants des usagers du déroulement de la prochaine campagne de pâturage. Le POPI débouche également sur l'élaboration d'un règlement pastoral intercommunal approuvé et cosigné par les élus et les éleveurs (Thavaud Pascal, 2013). Le POPI, outil de concertation visant à dynamiser le pastoralisme à l'échelle local, a connu un certain succès: celui du Pays de Fayence, localisé entre la Dracénie et le Pays de Grasse, est en cours de construction.

La prise en compte du pastoralisme dans la gestion des rapports fonciers se réalise surtout en amont du zonage: schémas d'aménagement, projets de territoires (telles que

\section{Plan d'Occupation Pastoral Intercommunal,}

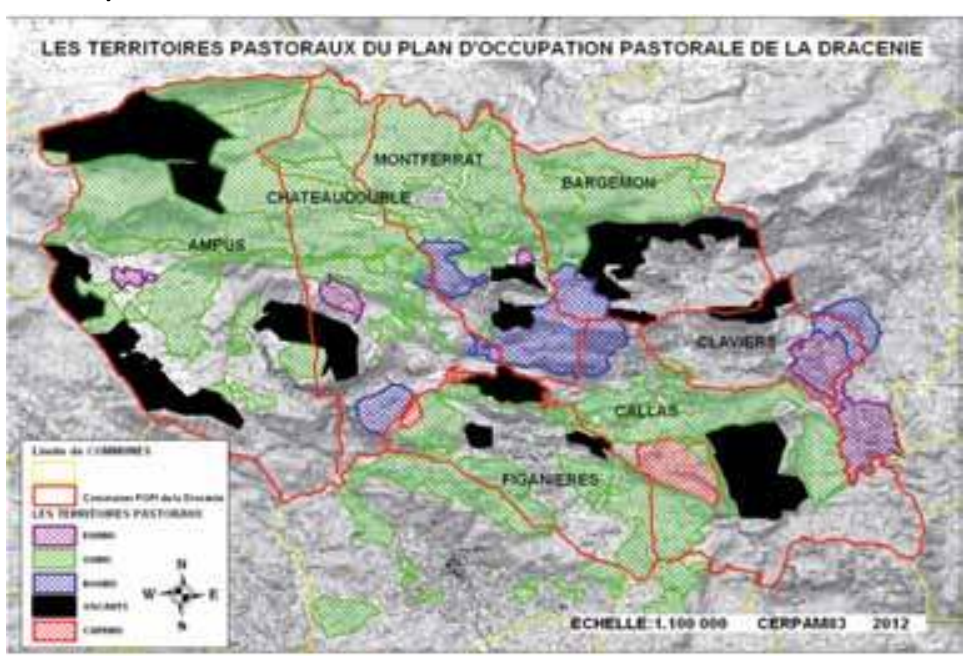

charte foncière du département de Haute-Provence du 23 avril 2010 par exemple, ou les chartes portant sur l'agriculture durable, comme celle du « Pays A3V pour une agriculture vivante, dynamique et porteuse d'avenir, 2010-2014», ainsi que la charte du Parc national des (évennes) et politique foncière de promotion de l'élevage, comme les opérations concertées d'aménagement et de gestion des espaces ruraux en pays viganais (OCAGER, devenues Terra Rural) et les aménagements fonciers (voir les opérations groupées d'aménagement foncier). Elle se traduit sur fond de pression foncière de plus en plus accentuée 
selon les territoires. Enfin, depuis peu apparaissent des planifications pastorales territorialisées à travers le « Plan Pastoral Territorial » véritable outil pour la mise en valeur pastorale des espaces, à destination des agriculteurs réunis dans un collectif, des collectivités territoriales et des associations. L'exemple du Plan pastoral des Monts d'Ardèche (2011-2016) est significatif d'une volonté politique: « le Plan Pastoral Territorial est un outil proposé par la région Rhône-Alpes pour soutenir les pratiques pastorales extensives et pour mettre en valeur les espaces pastoraux. Ce plan a pour but, à partir d'une concertation de l'ensemble des acteurs et usagers du territoire, d'instaurer un programme pluriannuel d'actions bénéficiant de crédits à l'amélioration pastorale (équipements, gestion du foncier, conditions de travail, actions agro- environnementales, multi-usages des espaces...) » (Grivel \& Bonin, 2011, 1). En effet, programmé pour cinq ans, le plan est cofinancé par l'Union européenne et le département de l'Ardèche. Il intéresse 1100 élevages (dont $65 \%$ non professionnels) et porte sur 34000 brebis, 13300 bovins et 8000 chèvres. Ce Plan pastoral procède par deux étapes opérationnelles:

a) une « structuration du foncier pastoral pour répondre au problème du morcellement et rechercher des solutions pour sécuriser l'utilisation du foncier » par la création d'associations foncières pastorales, la signature de conventions pluriannuelles de pâturage, la « sensibilisation des propriétaires et des élus locaux », et l'appui à la création de groupements pastoraux;

b) le soutien de projets d'ouverture des milieux et la lutte contre l'embroussaillement, un appui technique pour une gestion pastorale propice au maintien des milieux ouverts, et des aides à l'investissement matériel (clôture, pistes, cabane de bergers, ...).

Du passé au présent, le foncier dédié au pastoralisme a subi l'enjeu de la libération des fonds (le fait qu'aucun droit d'usage ne vienne limiter le droit de propriété) puis une reconnaissance au nom du territoire, des enjeux écologiques et culturels. Le régime de la propriété foncière exclusive et relativement absolue de 1804 qui n’a eu de cesse de défendre un usage individualiste de l'espace se confronte de nos jours aux « projets de territoire » qui émergent des documents de planification, d'urbanisme et d'organisation de l'espace. L'ère d'un renouveau dans le rapport au fonds en termes de « territoire » émerge. La conservation environnementale des Parcs nationaux, par exemple, entre dans une démarche socio-écologique par la « mise en adhésion» des populations à une charte (cf. loi n² 2006-436 du 14 avril 2006 relative aux parcs nationaux, aux parcs naturels marins et aux parcs naturels régionaux). La volonté du législateur de maintenir les paysages, par l'adoption d'une loi spécifique le 8 janvier 1993, suivi de la convention européenne du paysage (Florence, 20 octobre 2000), conduit les élus dans les plans locaux d'urbanisme et dans le cadre des parcs naturels régionaux, à intégrer les structures paysagères dans la façon de penser le territoire. Le fonds est visé directement dans ce que le propriétaire public ou privé peut en faire. Les acteurs qui le façonnent comme les éleveurs avec leur troupeau s'intègrent dans une dimension d'intérêt général par l'expression de valeurs qui s'imposent à la propriété privée individuelle. 


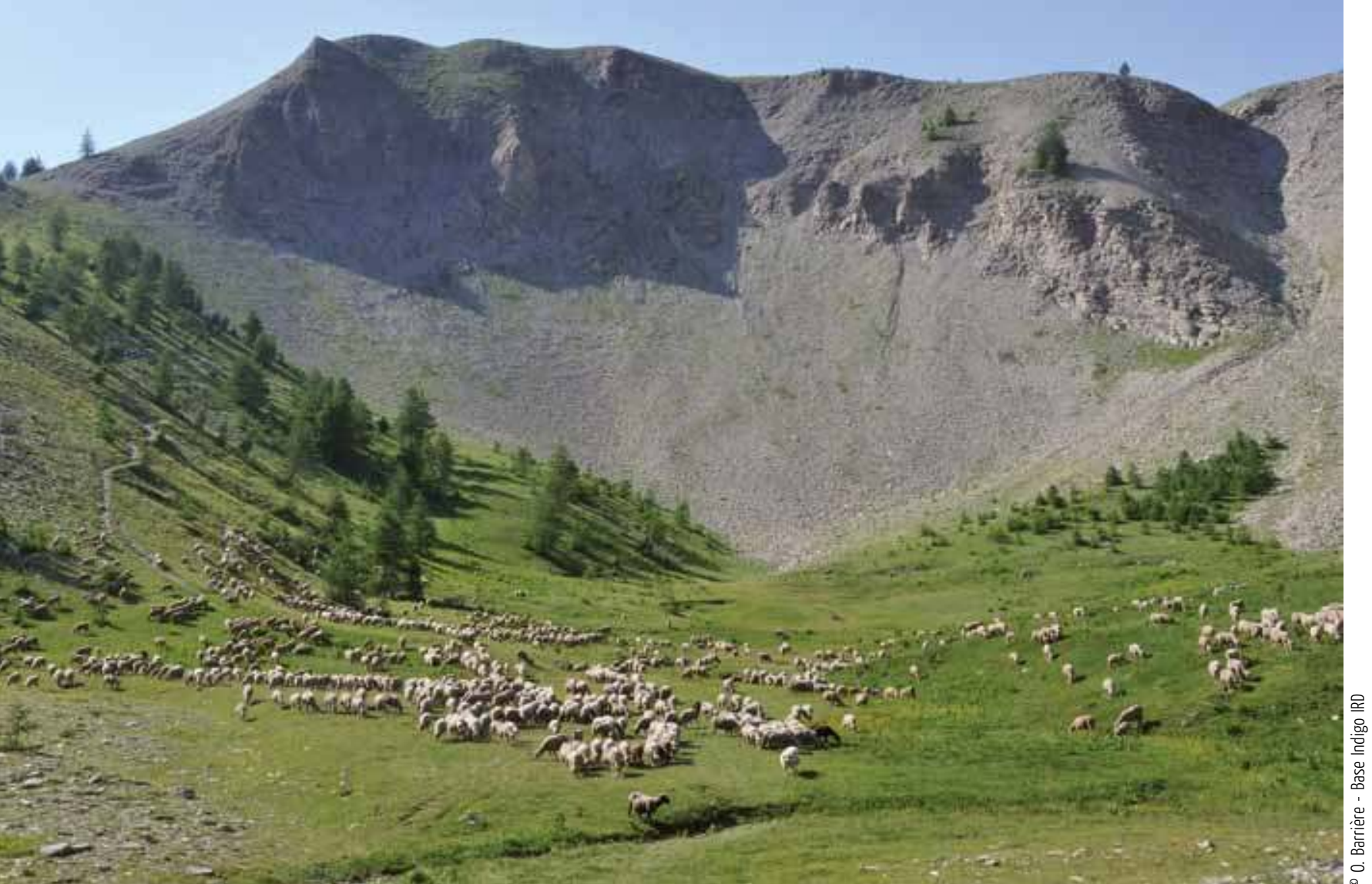

\section{Alpes de Haute Provence, Mont Pelat \\ Près d'Allos troupeau en estive gardé par un berger salarié, juillet 2012. \\ Le droit de pâture, un enjeu qui acquiert une « valeur universelle »}

« On veille à préserver nos zones agricoles », nous affirme un élu de la commune de Beauvezer (04), en juin 2012 lors d'un entretien. L'enjeu pour les politiques publiques est de trouver les façons de se dégager de l'emprise du régime de la propriété privée pour maintenir une multifonctionnalité et une mobilité dans l'espace afin de conserver l'activité pastorale, voire de la développer.

La question du régime foncier (le rapport juridique à la terre) face à celle de l'identité territoriale (ce qui fait territoire) nécessite d'être pris en charge dans la gouvernance et l'administration des territoires. Tout d'abord, en raison du paradigme qui sous-tend chaque identité locale et qui n'est donc pas toujours le même. L'ordre étatique défendant une relation de propriété à l'espace et situé sous le joug du principe de l'unité nationale, se superpose davantage aux enjeux locaux du territoire, dont la consistance repose sur une relation de valeurs partagées, qu'il ne s'y fond. Ensuite, en raison de l'exclusivité foncière qui entre de plus en plus en contradiction avec une gestion collective des ressources du territoire. Pour les élus et les gestionnaires qui adoptent et qui mettant en œuvre les politiques publiques, l'enjeu pour eux est de contourner la butte du régime de la propriété foncière. La négociation avec les propriétaires devient un défi quand il s'agit de maintenir et de développer le pastoralisme: des relations sont à organiser entre propriétaires et éleveurs. 


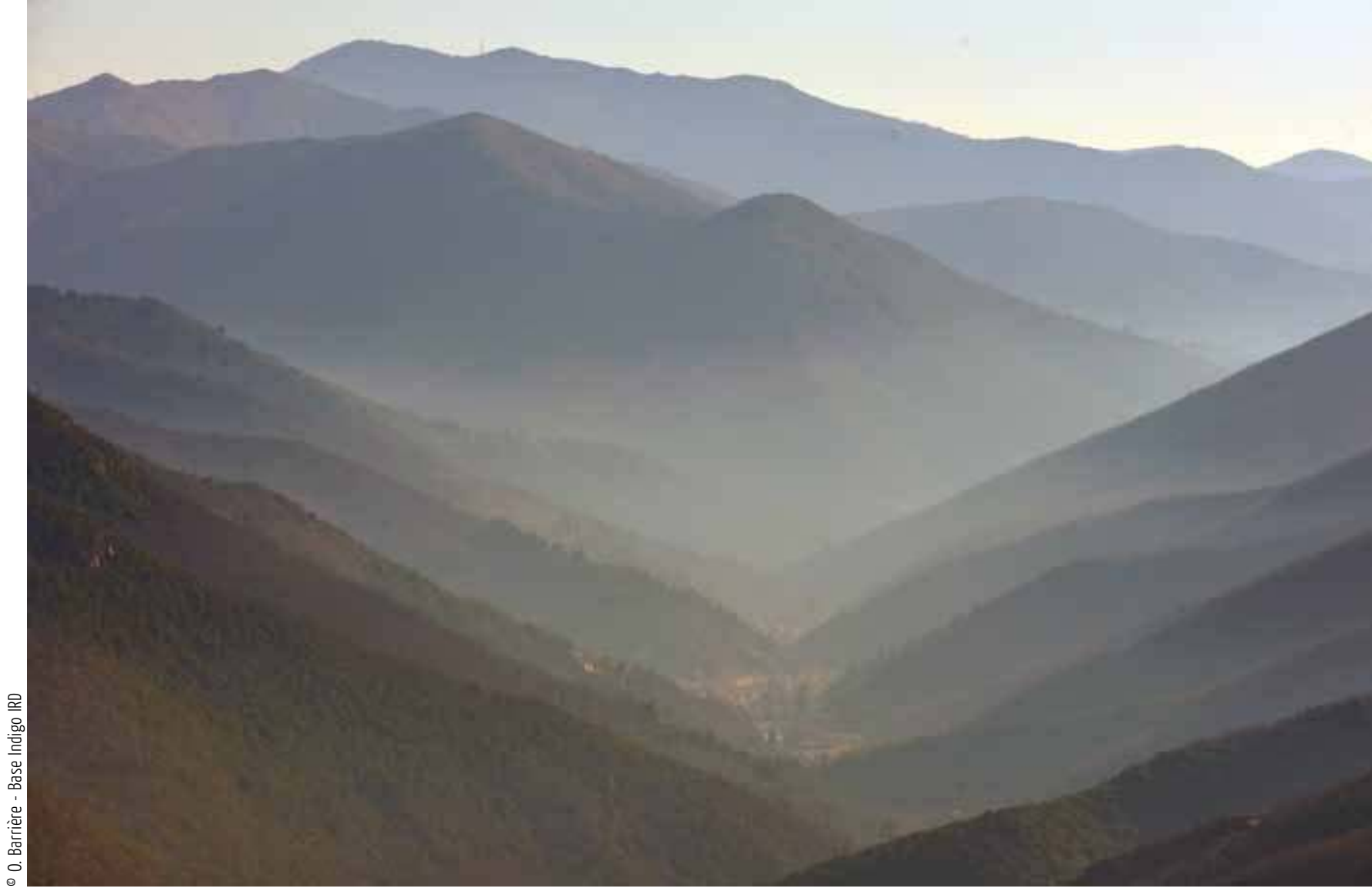

Dans les Cévennes, l'accès au foncier et sa sécurisation (par contrats écrits) sont Vallée cévenole près du Mont Aigoual Couché du soleil, vue sur des pâturages fruitiers sous bois (châtaignes et glands). ressentis et décrits par les éleveurs comme constituant la contrainte principale pouvant mettre en péril l'installation ou la confortation d'une exploitation pastorale. Parmi les solutions préconisées par eux, il est nécessaire de reconsidérer le droit d'usage face à l'appropriation privative de l'espace. L'exclusivité du droit de propriété peut en effet aboutir à des situations jugées injustes et incohérentes dans le contexte actuel de la raréfaction des terres agricoles: un éleveur est privé de son droit d'usage (pâturer une parcelle) tandis que le propriétaire pratique une rétention foncière et laisse « se dégrader » sa propriété, ou du moins non utilisée. Reconnaître la légitimité des droits d'usage liés aux nécessités pastorales (accès, passage, pâturage, etc.) contribuerait alors à faire vivre le pastoralisme en dépassant les inégalités économiques et financières générées par le paradigme de la propriété privée. De nouvelles valeurs sont convoquées par la reconnaissance du droit d'usage car l'usage local obéit à une logique collective servant les intérêts du territoire et de l'ensemble de ses habitants. Une des raisons d'être de la protection des droits d'usage pastoraux face au droit de propriété serait alors le maintien et le développement d'un territoire dont l'identité repose fortement sur les pratiques pastorales.

Face au rapport de force entretenu entre l'appropriation privative exclusive d'un espace et la reconnaissance des usages pratiqués sur cet espace, des alternatives semblent se dessiner et offrent une vision différente des rapports fonciers et de gestion des ressources foncières et environnementales. Lassociation Terre de Liens est par exemple devenue un acteur foncier majeur dans le monde agricole en ce qu'elle propose une vision innovante de l'installation et de la transmission des exploitations en même temps qu'un autre rapport 
à la terre, collectif et solidaire. C'est l'appropriation collective et solidaire du foncier agricole qui génère ici la protection du droit d'usage d'une terre contre l'exclusivité de la propriété privée. Autre exemple, le Groupement Foncier Agricole (GFA), qui est une société civile immobilière (SCI) particulière au domaine agricole, permet « d'acquérir et de gérer collectivement une propriété agricole (propriété bâtie ou foncier agricole) ». Il peut être bailleur et louer ses terres à un exploitant ou exploiter lui-même la terre en « faire valoir direct». Ici, c'est encore l'usage agricole qui est favorisé en développant, auprès des propriétaires privés, une conception collective et solidaire de l'accès à la terre.

Les limites qu'impose le régime du droit de propriété deviennent des contraintes à la gestion de l'espace, et à une approche collective de l'usage des ressources environnementales. Les valeurs de solidarité dans les projets de territoire buttent devant la mosaïque des portions de terres, devant la multiplicité des propriétés qui se transmettent en se fractionnant, restent en indivision, ou encore celles abandonnées par des héritiers inconnus ou disparus. Or, l'enjeu pastoral entre dans le jeu paysager. La mosaïque des parcelles, closes ou non, impose un ordre que les troupeaux remettent en question par leur mobilité à travers le territoire. Et, là où ces derniers ne passent pas les lieux se referment sur eux-mêmes, la forêt reprenant le dessus en se densifiant par la suppression progressive ou la réduction des voies de passage. Ainsi, le système de la plénitude des pouvoirs sur une portion d'espace s'oppose à une multifonctionnalité construisant le territoire qui lui, est bien commun. Ce que reconnaît justement l'Unesco par le classement des paysages agropastoraux en patrimoine mondial en leurs conférant une valeur universelle à la culture vivante qu'ils expriment.

Le récent classement, en juin 2011, des Causses et Cévennes comme « paysage culturel de l'agro-pastoralisme méditerranéen » au patrimoine mondial de l'Unesco (dans la catégorie " paysages culturels évolutifs vivants »), de la convention du patrimoine mondial, naturel et culturel (Paris, 1972) et de la Déclaration de valeur universelle exceptionnelle (2008), souligne les enjeux liés au maintien de l'activité pastorale.

Les acteurs locaux, dont les élus, prennent petit à petit conscience qu'il est nécessaire de maintenir les usages pastoraux qui ont façonné les paysages afin de préserver l'intégrité de ces paysages contre les risques de fermeture, d'embroussaillement et de disparition des éléments culturels du pastoralisme imprimant l'espace. Si les éleveurs venaient à disparaître et leurs pratiques pastorales avec, pendant combien de temps les paysages agro-pastoraux continueront-ils d'exister? Aussi, la préservation du broutage sur un territoire où les éleveurs sont de moins en moins nombreux et la reprise ou l'installation de jeunes éleveurs appelle la mise en place d'une politique pro pastorale de préservation du foncier pastoral.

Une expérience est en cours à ce sujet. La communauté de communes Causses Aigoual Cévennes Terres solidaire se mobilise en élaborant un pacte pastoral intercommunal, conférant une valeur identitaire territoriale à la pâture. Ladoption du Pacte (par délibération de l'établissement public de coopération intercommunal) est prévue lors du prochain Conseil communautaire en juin 2015. Un concept phare du Pacte est celui d'une servitude pastorale déclarée d'intérêt collectif. Elle ne peut être imposée aux propriétaires récalcitrants mais négociée. Dépasser les contraintes du régime de la propriété ne demeure pas impossible mais nécessite la hardiesse d'innovations depuis l'abolition de la « vaine pâture » en 1889 (sauf dans les rares communes françaises qui ont souhaité expressément la conserver). La servitude pastorale territoriale confère le libre passage et broutage sur tout fonds non clos des troupeaux sans que ceux-ci ne causent de préjudice dans le but de maintenir la mobilité des troupeaux dans le territoire. Cette servitude de passage et de broutage pastorale dans l'intérêt 
public correspond à la « tolérance de passage » des usages locaux identifiés par le recueil officiel des usages locaux du département du Gard datant de 1963. Ce droit de pâture définit un droit d'usage pastoral susceptible de n'être limité soit par une mise en défens dûment motivée par un risque certain de préjudice, soit par un refus catégorique non négociable par le propriétaire. C'est l'intérêt général qui justifie toutes les atteintes portées au droit de propriété et le pastoralisme est justement considéré comme d'intérêt général par le législateur français (art. L113-1 \& L113-2 du code rural). La pâture sort ainsi de la marginalisation des pratiques modernes par le droit, lui-même qui l'avait bien condamné en 1889.

L'histoire du droit foncier pastoral mise en parallèle avec celui de la propriété foncière apporte un éclairage sur la valeur et l'intérêt que toute société confère à l'activité pastorale et aux pâturages marqueurs des paysages et des territoires. Les relations à l'espace s'élaborent sur des convictions, des croyances, des paradigmes que la régulation juridique formalise sous forme de règles, de principes et de régimes. Deux statuts patrimoniaux tentent de cohabiter à travers la notion de «patrimoine personnel » (relevant du code civil) et celui de "patrimoine commun ». L'entre-deux serait une perspective. Mais, «bien que la plupart des systèmes pastoraux reposent sur des terres communautaires, la mise en place de titres de propriété fonciers individuels peut permettre d'établir des systèmes pastoraux productifs et durables. Cependant, les exemples de conversions réussies de terres communautaires en régimes fonciers individuels sont rares ", voire très rares ou exceptionnels. Ce constat du Secrétariat de la Convention sur la diversité biologique (2010) est l'aveu même de l'impasse de privilégier le régime foncier individuel comme une voie inéluctable, et qui ne l'est pas.

Ce travail a été conduit dans le cadre du projet de recherches MOUVE financé par l'Agence nationale de la recherche (projet ANR-2010-STRA-005-01).

\section{NOTES}

Photo d'ouverture: Entre bergers, tirage au sort des imizar (cabanes) du pâturage d'altitude (agdal) « Marat » dans le territoire Ait Zekri sur le versant sud du Haut Atlas marocain. L'attribution de l'amazir (cabane) conditionne l'accès aux pâturages alentours. Elle est au cœur de la stratégie pastorale du berger. mai 2005, Photo : Olivier Barrière, Base Indigo IRD.

1. Le terme « herbe » doit être compris globalement comme la ressource fourragère qui ne se limite pas à la strate herbacée mais aussi ligneuse.

2. Loi « publiée selon la procédure d'urgence et exécutée comme loi de l'État». Voir sur le droit pastoral Ly Ibrahima, 2004 et Mekouar Ali, 1998

3. «L'enjeu est de promouvoir des démarches de développement territorial, économique et social qui soient davantage intégrées et plus constructives au regard des capacités et des attentes de ces sociétés d'éleveurs et dans le respect de la durabilité des milieux fragiles et uniques qu'elles habitent » (Bonnemaire \& Jest, 2013).
4. La production herbagère est spontanée ou non, c'est à dire avec ou sans l'intervention directe de l'homme qui, soit ne laboure pas, ne sème pas, on parle alors de prairie permanente, soit opère une rupture en labourant, on parle ainsi de prairie non permanente.

5. Par exemple, l'emprise par le sillon de l'agriculteur génère, encore souvent en Afrique, un droit foncier que ne confère pas le broutage des animaux.

6. Le droit de panage est le droit de faire pâturer des porcs en forêt pour y consommer les fruits des arbres forestiers 


\section{POUR CITER CET ARTICLE}

Barrière, O. 2015 La Mise en patrimoine de la pâture par le droit. Entre marginalisation et valeur universelle. In Dupré, L., Lasseur, J. \& R. Poccard-Chapuis Pâturages Techniques E Culture 63 : 182-201.

\section{RÉEÉRENCES}

Bonnemaire, J. \& Corneille J. (Dir.) 2013 Le Pastoralisme en Haute-Asie: la raison nomade dans l'étau des modernisations, Études mongoles et sibériennes centreasiatiques et tibétaines (43-44). [En ligne], consulté le 09 décembre 2014. URL : http://emscat.revues.org/1995.

Devienne, S. 2013 Régulation de l'accès aux parcours et évolution des systèmes pastoraux en Mongolie in Bonnemaire, J. \& Corneille J. (Dir.) 2013 Le Pastoralisme en Haute-Asie: la raison nomade dans l'étau des modernisations, Études mongoles et sibériennes centreasiatiques et tibétaines (43-44) [En ligne], consulté le 09 décembre 2014. URL : http://emscat.revues.org/2104 ; DOI : 10.4000/emscat.2104.

Fernandez-Gimenez, M. - E. \& Batbuyan, B. 2004 Law and Disorder: Local Implementation of Mongolia's Land Law, Development and change, (35) 1: 141-166.

Le Roy, E. 2011 La Terre de l'autre. Une anthropologie des régimes d'appropriation foncière. Paris: LGDJ

Grellier, B. 2006 Berger transhumant sur l'Aigoual : la transhumance ovine et les savoirs du berger, Revue internationale des sciences sociales 1 (187): 169-172.

Grivel, G. \& Bonin, R. 2011 Plan pastoral territorial des Monts d’Ardèche. Parc naturel régional des Monts d'Ardèche. (Diagnostic et programme d'actions 2011-2015).

Ibrahima, L. 2004 Essai de présentation des tendances d'évolution du droit pastoral en Afrique de l'ouest Burkina-Faso, Guinée, Mali, Mauritanie, Niger, Sénégal, FAO, Étude juridique 35. En ligne : <http:// www.fao.org/fileadmin/user_upload/legal/docs/lpo35f.pdf> consulté le 9 février 2015.

Meuret, M. \& al. 1995 Faire pâturer sur parcours, Renc. Rech. Ruminants (2) : 27-36.

Mounet C. \& Turquin O. 2014 Espaces et acteurs pastoraux: entre pastoralisme(s) et pastoralité(s), Journal of Alpine Research | Revue de géographie alpine 102 - 2 (mis en ligne le 24 mars 2014), consulté le 15 septembre 2014. <http://rga.revues.org/2462>.

Mekouar M. A. 1998 Pastoralisme et environnement en droit comparé sub-saharien. In Prieur, M. (Dir.) Les Hommes et l'environnement. Quels droits pour le vingt et unième siècle? Études en hommage à Alexandre Kiss. Paris: Frison Roche: 652-670.

Proudhon J.-B.-V. 1824 Traité des droits d'usufruit, d'usage, d'habitation et de superficie tome VI. Dijon: Victor Lagier Libraire.

Secrétariat de la Convention sur la diversité biologique, 2010 Pastoralisme, conservation de la nature et développement: un guide des bonnes pratiques. Montréal. En ligne : <http://www.cbd.int/development/ doc/cbd-good-practice-guide-pastoralism-booklet-web-fr.pdf>, consulté le 10 mars 2015

Thavaud P. 2013 Le Plan d'occupation pastorale, une démarche de coopération entre éleveurs et élus pour dynamiser le pastoralisme à l'échelle territoriale, Revue Pastum de l'association française de pastoralisme, 100: 5-9. 


\section{RÉSUMÉ}

La Mise en patrimoine de la pâture par le droit. Entre marginalisation et valeur universelle. La pâture et l'activité pastorale dépendent en partie de ce que le droit en fait. Les contextes socio-écologiques dans le monde sont très variés mais il se dégage deux grands paradigmes, celui de la propriété privée et celui de la patrimonialité commune ou collective. Le rapport entre ces deux systèmes conduit soit à pérenniser l'activité pastorale, soit à la contraindre ou à l'éliminer. La marginalisation du pastoralisme n'est pas forcément rédhibitoire. De plus, le droit international parvient à classer et élever au statut de valeur universelle le paysage agro-pastoral en tant que « culture vivante ».

\section{ABSTRACT}

Recognition of grazing heritage through legislation. Between marginalization and universal value. Grazing and pastoral activities depend in part on how the law deals with them. Socio-ecological contexts are extremely varied worldwide, but generally correspond to one of two major paradigms: that of private property or that of communal or collective property. The relationship between these two systems leads either to sustaining pastoral activities, limiting them or eliminating them. The marginalization of pastoralism is not necessarily a fatality. In fact, the «territorialization» of grazing land promotes the multifunctionality of activities with environmental issues through pastoral development. Moreover, international law - by means of the Convention for the Protection of the World Cultural and Natural Heritage and the Statement of Outstanding Universal Value - has made it possible to classify the agro-pastoral landscape as a «living culture» and to raise it to the status of a universal value.

\section{MOTS-CLÉS}

droit foncier, droit pastoral, pâture, patrimoine, territoire

\section{KEYWORDS}

property law, pastoral law, grazing, heritage, territory
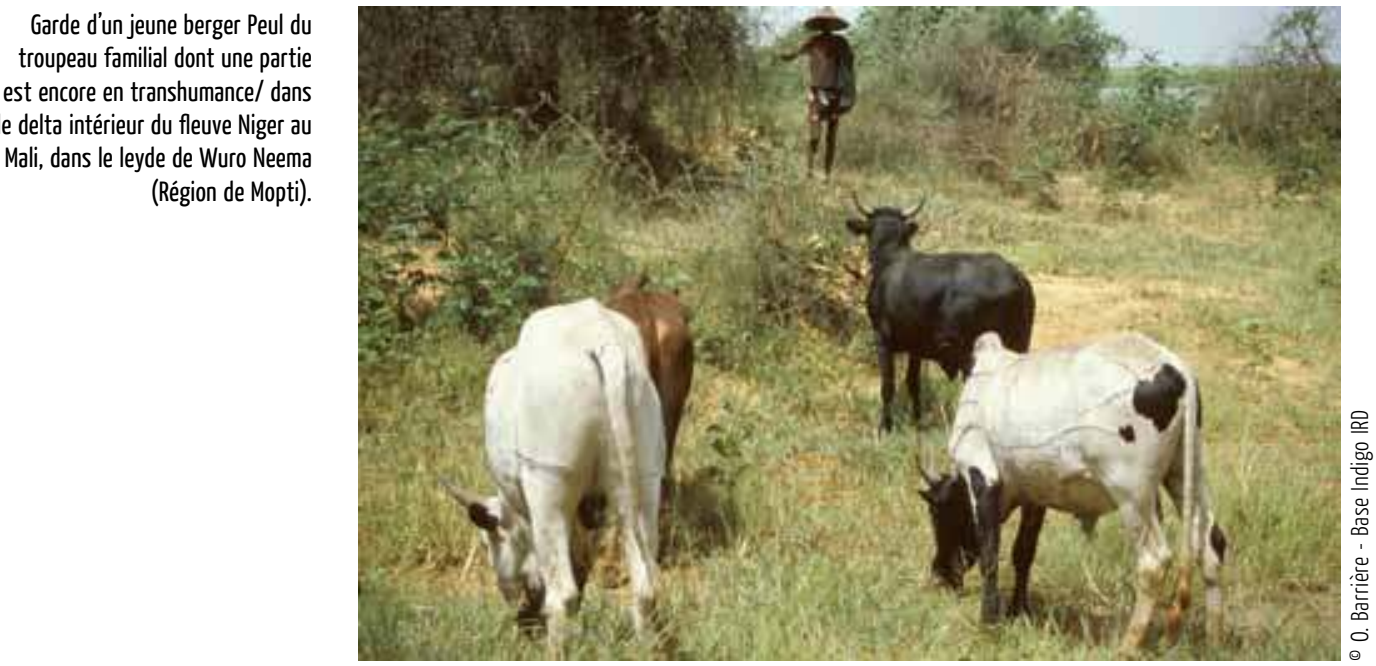